\title{
Coordinate-free Characterization of the Symmetry Classes of Elasticity Tensors
}

\author{
Andrej Bóna • Ioan Bucataru • Michael A. Slawinski
}

Published online: 10 July 2007

(C) Springer Science + Business Media B.V. 2007

1 Journal of Elasticity Volume 87(2-3) 109-132, doi: 10.1007/s10659-007-9099-z

1. Equation (5): there should be a factor of 2 in front of $c_{-} 45$.

The correct text should be as follows:

$$
C(e)=\left[\begin{array}{cccccc}
c_{11} & c_{12} & c_{13} & \sqrt{2} c_{14} & \sqrt{2} c_{15} & \sqrt{2} c_{16} \\
c_{12} & c_{22} & c_{23} & \sqrt{2} c_{24} & \sqrt{2} c_{25} & \sqrt{2} c_{26} \\
c_{13} & c_{23} & c_{33} & \sqrt{2} c_{34} & \sqrt{2} c_{35} & \sqrt{2} c_{36} \\
\sqrt{2} c_{14} & \sqrt{2} c_{24} & \sqrt{2} c_{34} & 2 c_{44} & 2 c_{45} & 2 c_{46} \\
\sqrt{2} c_{15} & \sqrt{2} c_{25} & \sqrt{2} c_{35} & 2 c_{45} & 2 c_{55} & 2 c_{56} \\
\sqrt{2} c_{16} & \sqrt{2} c_{26} & \sqrt{2} c_{36} & 2 c_{46} & 2 c_{56} & 2 c_{66}
\end{array}\right]
$$

2. Within the paragraph before equation (34) "rotation by angle $\theta-\eta$ " should be as follows:

"rotation by angle $(\theta-\eta) / 3$ "

The online version of the original article can be found at http://dx.doi.org/10.1007/s10659-007-9099-z.

A. Bóna $\cdot$ M. A. Slawinski

Department of Earth Sciences, Memorial University, St. John's NL A1B 3X5, Canada

A. Bóna

e-mail: abona@mun.ca

M. A. Slawinski

e-mail: mslawins@mun.ca

I. Bucataru $(\bowtie)$

Faculty of Mathematics, “Al. I. Cuza” University, Iasi 700506, Romania

e-mail: bucataru@uaic.ro 
3. Equation (34): there should be a factor of 3 in the denominator of the argument of the trigonometric functions $(2 \theta+\eta)$.

The correct text should be as follows:

$$
\sigma=\|b\|\left[\begin{array}{ccc}
\gamma_{2} \cos ((2 \theta+\eta) / 3) & \gamma_{2} \sin ((2 \theta+\eta) / 3) & \sin ((2 \theta+\eta) / 3) \\
\gamma_{2} \sin ((2 \theta+\eta) / 3) & -\gamma_{2} \cos ((2 \theta+\eta) / 3) & \cos ((2 \theta+\eta) / 3) \\
\sin ((2 \theta+\eta) / 3) & \cos ((2 \theta+\eta) / 3) & 0
\end{array}\right]
$$

4. Equation (35): the denominator of the expression for c_44 should be multiplied by 2 . The correct text should be as follows:

$$
\begin{aligned}
& c_{11}=\frac{\left(\gamma_{1}+\gamma_{3}\right) \gamma_{2}^{2} \gamma_{1}^{2}+\left(\gamma_{1}+\gamma_{4}\right) \gamma_{1}^{2}+2\left(\gamma_{2}+\gamma_{3}\right) \gamma_{2}^{2}+2\left(\gamma_{2}+\gamma_{4}\right)}{2\left(2+\gamma_{1}^{2}\right)\left(1+\gamma_{2}^{2}\right)}, \\
& c_{12}=\frac{\left(\gamma_{1}-\gamma_{3}\right) \gamma_{2}^{2} \gamma_{1}^{2}+\left(\gamma_{1}-\gamma_{4}\right) \gamma_{1}^{2}+2\left(\gamma_{2}-\gamma_{3}\right) \gamma_{2}^{2}+2\left(\gamma_{2}-\gamma_{4}\right)}{2\left(2+\gamma_{1}^{2}\right)\left(1+\gamma_{2}^{2}\right)}, \\
& c_{13}=\frac{\left(\gamma_{2}-\gamma_{1}\right) \gamma_{1}}{2+\gamma_{1}^{2}}, c_{33}=\frac{2 \gamma_{1}+\gamma_{2} \gamma_{1}^{2}}{2+\gamma_{1}^{2}}, \\
& c_{14}=\frac{\left(\gamma_{3}-\gamma_{4}\right) \gamma_{2}}{2\left(1+\gamma_{2}^{2}\right)}, c_{44}=\frac{\gamma_{3}+\gamma_{4} \gamma_{2}^{2}}{2\left(1+\gamma_{2}^{2}\right)},
\end{aligned}
$$

